\title{
Target frequency modulates object-based attention
}

\author{
Joseph C. Nah ${ }^{1} \cdot$ Sarah Shomstein ${ }^{2}$
}

Published online: 3 June 2020

(C) The Psychonomic Society, Inc. 2020

\begin{abstract}
Decades of research have provided evidence that object representations contribute to attentional selection. However, most evidence for object-based attentional allocation is drawn from studies employing the two-rectangle paradigm where the target distribution is biased towards the cued object. It is thus unclear whether object-based attentional selection is from object representations or a consequence of spatial attention based on statistical imbalances. Here, we investigate the extent to which target frequency modulates object-based attention by systematically manipulating the frequency of target appearance in a particular spatial location within objects to equate spatial allocation, bias specific spatial locations, or bias objects. In four experiments, participants were presented with a variant of the two-rectangle paradigm in which one end of a rectangle was cued and performed a target discrimination task. Critically, the target location probabilities were parametrically manipulated. The target could appear equally in all ends within the objects (valid, invalid within-object, invalid between-object, diagonal) (Experiment 1) or with overall equality between objects but biased towards the invalid locations (Experiment 2). The target could also appear in three locations (valid, invalid within-object, invalid between-object) distributed equally between objects but biased towards the invalid between-object location (Experiment 3) or with an overall bias towards the invalid between-object location (Experiment 4). We observed that while objects bias attention, spatial biases are prioritized over object representations. Combined results suggest that object-based contribution to attentional guidance is the result of both spatial probabilities and object representations.
\end{abstract}

Keywords Object-based attention $\cdot$ Space-based attention $\cdot$ Visual selective attention

\section{Introduction}

Visual selective attention is the cognitive mechanism through which a subset of the overwhelming visual information is selected for further processing. While decades of research demonstrated that attention is constrained by spatial locations (Carrasco, 2011; Eriksen \& Eriksen, 1974; Posner, 1980; Posner, Snyder, \& Davidson, 1980), other dimensions that exist within space also guide attentional selection. For instance, scenes in the real-world contain many disparate objects and experimental evidence supports the observation that objectbased representations contribute to attentional allocation

Joseph C. Nah

josephcnah@gmail.com

1 Center for Mind and Brain, University of California, Davis, CA 95618, USA

2 Department of Psychology, The George Washington University, Washington, DC, USA
(Duncan, 1984; Rock \& Gutman, 1981). In a study conducted by Duncan (1984), participants attended to two spatially superimposed objects (a rectangle and a slanted line) and were tasked to report two features from the display. The rectangle was tall or short with a small gap on the left or right; the line was dotted or dashed and slanted to either the left or the right. Participants were significantly more accurate when reporting two features from one object (e.g., rectangle size and gap location) than when reporting one feature from each object (e.g., line direction and rectangle size). Since both objects were occupying the same spatial location, this result serves as evidence that attention is constrained by object representations.

However, while Duncan's study demonstrates an objectbased attentional benefit, considering that no two objects can truly occupy the same space, it was pointed out by Posner and noted by Duncan (see Footnote 3 in Duncan, 1984) that the two objects were inferred to differ in depth (Egly, Driver, \& Rafal, 1994; Watt, 1988). To tease apart this spatial confound from object influence, the two-rectangle paradigm (Egly et al., 1994) was developed, from which much of the evidence in support of object-based attentional selection has been 
garnered. In the two-rectangle paradigm, participants are presented with two identical parallel rectangles oriented either horizontally or vertically. Following a brief delay, one end of the rectangle is exogenously cued to engage attention. After another short delay, a target is presented in one of three locations: the cued location (valid), the opposite end of the cued rectangle (invalid same-object), or at the equidistant location in the non-cued rectangle (invalid different-object). Responses to targets in the valid location are fastest, a finding that serves as evidence for space-based attentional guidance. The intriguing finding from this paradigm, however, is that responses to targets that appear in the invalid same-object location are faster and more accurate than to targets in the invalid different-object location, albeit both being spatially equidistant from the cue. This difference is evidence of object-based attentional guidance (i.e., object-based effect), as object boundary is the only difference between the two invalidly cued locations. This object-based effect is robust, having been replicated by numerous studies over two decades (Behrmann, Zemel, \& Mozer, 1998; Moore, Yantis, \& Vaughan, 1998; Müller \& Kleinschmidt, 2003; Shomstein \& Behrmann, 2006, 2008; Shomstein \& Yantis, 2002; Theeuwes, Mathot, \& Kingstone, 2010; Watson \& Kramer, 1999).

Despite much research on the influence of object representations on attentional selection, there remains an alternative explanation. A major concern is that most object-based paradigms included an implicit bias that favored locations within the cued object. In the two-rectangle paradigm, the target distribution is always heavily biased towards the cued object. For instance, in the original Egly et al. (1994) study, on targetpresent trials, $75 \%$ of the trials appear within the cued object ( $50 \%$ valid, $25 \%$ invalid same-object). This bias is the result of the high percentage of valid trials, necessary to observe an object-based effect and is present in most object-based attention studies using the two-rectangle paradigm (e.g., Avrahami, 1999; Kravitz \& Behrmann, 2011; Lamy \& Egeth, 2002; Marino \& Scholl, 2005; Nah, Neppi-Modona, Strother, Behrmann, \& Shomstein, 2018; Robertson \& Kim, 1999). Thus, this bias towards the cued object makes it difficult to ascertain whether an object-based effect is purely the result of object representation, or whether the object benefit is a direct result of target contingency.

It is a well-established fact that target contingency is a strong cue to attentional selection (Geng \& Behrmann, 2002). While some studies investigated how target contingency modulates attention and how it interacts with object-based attention (Shomstein \& Behrmann, 2008; Shomstein \& Yantis, 2004), there was still an imbalance in target appearance between objects. Partially, this lingering imbalance continued to be present in most object-based paradigms because it was not a central focus of investigation. For instance, Shomstein and Yantis (2004) manipulated the probability of target appearance in the two invalid conditions. One of the two invalid locations could either be the high-probability location in which $41.7 \%$ of trials would appear. The other invalid location was the low-probability location in which $8.3 \%$ of trials would appear. The remaining 50\% was allocated to the valid location. Thus, depending on where the high-probability location was allocated, the target could appear within the cued object either $58.3 \%$ or $91.7 \%$ of the time, still carrying over an object imbalance.

By manipulating cue validity to a specific spatial location or to a specific object, a recent study dissociated locationbased cues and object-based cues to understand the role of object configuration in attentional allocation (Chou \& Yeh, 2018). Using a variant of the two-rectangle paradigm, the cue-target contingency was manipulated across a set of experiments with the goal of testing how the probability of target presentation at a specific cued location (location-based cues) and the probability of target presentation at a specific cued object (object-based cues) can influence attentional deployment. While the results suggested that when targets are biased towards the cued-object, informative location-based cues induced a spatial cueing effect and informative object-based cues led to an object-based effect, whether the attentional benefit of the cued object is the result of this spatial statistical imbalance remains unclear. Namely, participants may have anticipated the target appearing within the cued object, resulting in faster and more accurate allocation of attention within an object than between. This possible explanation questions whether an object-based effect is influenced by object representation and, by extension, challenges current mechanisms of object-based attention. In fact, recent studies that examine object-based attention in the absence of a spatial cue (and thus fully removing cued object bias), fail to find evidence of object influence on attentional guidance (Donovan, Pratt, \& Shomstein, 2017).

In this study, we investigate how spatial statistical imbalance interacts with object representations through the systematic manipulation of target frequency within the two-rectangle paradigm. Experiment 1 examined whether object representations guide attention when target appearance is perfectly balanced across all spatial locations and objects. In Experiment 2, while overall target frequency was balanced across objects (i.e., targets appeared equally on both objects), targets were equally biased towards the invalid same-object and invalid different-object locations over the valid and diagonal locations. In Experiment 3, target frequency was again balanced between the two objects, but biased towards the invalid different-object location over the valid and invalid sameobject locations. Lastly, in Experiment 4, target frequency was biased towards the invalid different-object location over the valid and invalid same-object locations. If object representations automatically constrain attention, there should always be a benefit when targets appear within the cued object, 
regardless of a spatial bias towards or against the cued object. If, however, the object-based effect is elicited by spatial statistical imbalance, attentional allocation should be more flexible. More specifically, attentional benefit should not adhere to object boundaries, but towards a specific biased location. Yet another possibility, one that we argue is more likely and bears out in our data, is that object-based effects are the result of the interaction of spatial probabilities and object contributions and not solely driven by one type of information.

\section{Method}

Participants A post hoc power analysis using the G*Power program (Faul, Erdfelder, Lang, \& Buchner, 2007) was conducted after data collection to demonstrate adequate power. Using a $\eta^{2}$ p of .56 taken from a previous study (Nah et al., 2018), the power analysis revealed that a sample size of $n=17$ was sufficient to achieve the power of .999. A total of 90 undergraduate students from The George Washington University (Experiment 1: $n=20$, mean age $=19.23$ years, range $=18-22$, one male; Experiment $2: n=26$, mean age $=$ 19.50 years, range $=18-24$, ten male; Experiment 3: $n=19$, mean age $=19.50$ years, range $=18-21$, five male; Experiment 4: $n=25$, mean age $=19.24$ years, range $=18$ 24, 11 male) participated in exchange for course credit. Each participant only took part in one of the experiments. All participants gave informed consent according to The George Washington University's institutional review board, were naïve to the experiment's purpose, and reported normal or corrected-to-normal vision.

Apparatus and stimuli The experiment took place in a dimly illuminated room with a monitor placed approximately $60 \mathrm{~cm}$ from the participant. The objects were black-outlined rectangles $\left(4.2^{\circ} \times 1.2^{\circ}\right)$ with a $4.2^{\circ}$ distance between the midpoints of the rectangles. The targets and distractors were $.59^{\circ} \times .59^{\circ}$ in size and all stimuli were presented on a gray background. Each end of each rectangle was equidistant from each other as well as the cue. A fixation cross $\left(0.3^{\circ} \times 0.3^{\circ}\right)$ was present throughout the experiment in the center of the screen.

Design and procedure Each trial started with two rectangles appearing for 1,000 ms (Fig. 1A). With the exception of Experiment 1, the object orientation (vertical, horizontal) was counterbalanced across participants (in Experiment 1, participants were presented with an equal number of horizontal or vertical rectangle trials). Next, a red cue randomly but equiprobably highlighted one end of the rectangle for $100 \mathrm{~ms}$. After a 350-ms inter-stimulus interval (ISI), a target letter " $\mathrm{T}$ " or "L" embedded among non-letter T/L hybrid distractors were presented within the remaining ends of the rectangle. The 350-ms ISI was used so that participants had enough time to apply the learned contingencies, or probability information (Drummond \& Shomstein, 2010; Shomstein \& Yantis, 2004). Participants performed a target identification task without breaking fixation. The display was present for 3,000 ms or until a response was made. The target could appear in the same location as the cue (valid), or in one of three invalid locations: at the opposite end of the cued object (invalid same-object), at the end of the uncued object nearest the cue (invalid different-object), or the opposite end of the uncued object (diagonal). Targets appearing in the invalid sameobject and invalid different-object condition were equidistant from the cue and targets appearing in the diagonal condition was furthest away. The diagonal condition was only in Experiments 1 and 2 (Fig. 1B). At the end of the experiment, participants completed a two-question survey asking whether they were aware of a cue-target contingency: (1) Did you notice anything about the frequency of where the target appears? (2) If you were told that there was an imbalance in where the target appeared, what do you think it would be?

Target contingency The cue-target contingency varied for each experiment (Fig. 1B). In Experiment 1, the target appeared in all four locations with an equal likelihood of $25 \%$ (ten blocks of 64 trials each). In Experiment 2, valid and diagonal trials comprised $10 \%$ of the total trials each with the remaining $80 \%$ equally divided among the invalid sameobject and invalid different-object conditions (ten blocks of 80 trials). In Experiment 3, 50\% of the trials were allocated to the invalid different-object trials with the remaining half equally divided among the valid and invalid same-object trials (seven blocks of 96 trials). Lastly, in Experiment 4, valid trials comprised $25 \%$ of the total trials, invalid same-object trials $15 \%$, and $60 \%$ were allocated to the invalid different-object trials (11 blocks of 80 trials).

\section{Results and discussion}

For all experiments, participants with an overall accuracy less than $90 \%$ were removed. Response times (RTs) less than $150 \mathrm{~ms}$ (anticipatory responses) and greater than 2,000 ms were also removed from the analysis. For Experiments 2-4, responses from the first two blocks were removed from the analysis to ensure enough time to learn the cue-target relationship (there were no qualitative differences in any of the reported effects without removal of the first two blocks). Spacebased effect was assessed by comparing RTs and accuracy for the invalid condition (collapsed equidistant invalid sameobject and invalid different-object) with the valid and diagonal condition. Object-based effect was investigated by comparing RTs and accuracy of the two invalid conditions. Lastly, none of the participants in the current set of studies reported 
a

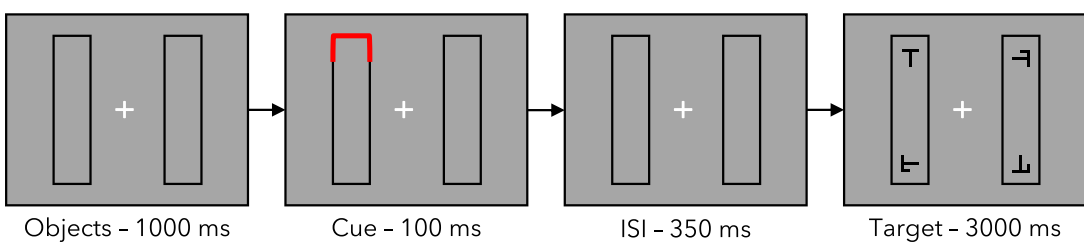

b

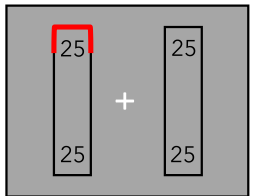

Experiment 1:

Spatial and Object Equality

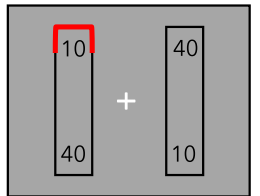

Experiment 2:

Spatial Bias (Invalid) and Object Equality

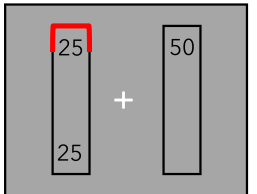

Experiment 3:

Object Equality and Spatial Bias towards

Different-Object

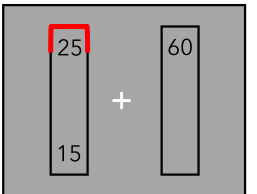

Experiment 4:

Spatial and Object Bias towards Different-Object

Fig. 1 Example trial sequence for all experiments (A), depicting a valid condition trial. Numbers within objects (B) represent target contingency to cue for each experiment in percentages. The location of the cue was random, but equiprobably distributed across the four possible locations

awareness of any relevant cue-target contingency based on the answers from the survey.

Experiment 1 - spatial and object equality Experiment 1 investigated whether object representations guide attention when no location or object is biased. No participants and .2\% of the trials were removed from the final analysis for a total of 20 participants. Space-based effects were examined by conducting a two-way repeated-measures ANOVA, with object orientation (horizontal, vertical) and spatial distance (valid, invalid, and diagonal) as within-subjects factors, and accuracy as a dependent measure. There was a significant main effect of spatial distance, $F(2,38)=4.18, p=.023, \eta_{p}^{2}=.18$, with planned $\mathrm{t}$ tests revealing that participants were more accurate in the valid $(M=97.25, S E=.52)$ than in the invalid condition $(M=95.68$, $S E=.53)[t(19)=2.62, p=.017$, Cohen's $d=.59]$ and diagonal condition $(M=95.37, S E=.53)[t(19)=2.48, p=.023$, Cohen's $d=.56$. No other main effects or interactions reached significance $(F \mathrm{~s}<4.11, p \mathrm{~s}>.05)$.

Applying the same analysis on RT revealed a significant main effect of spatial distance, $F(2,38)=22.85, \mathrm{p}<.001, \eta_{\mathrm{p}}^{2}$ $=.55$ (Fig. 2A). Participants were faster at responding in the valid $(M=579.83 \mathrm{~ms}, S E=4.80)$ than the invalid trials $(M=$ $605.78, S E=4.51),[t(19)=6.39, p<.001$, Cohen's $d=1.43]$ and the diagonal trials $(M=645.54, S E=9.20),[\mathrm{t}(19)=5.55$, $p<.001$, Cohen's $d=1.24]$. Participants were also significantly faster at responding in the invalid than in the diagonal trials, $t(19)=3.48, p=.008$, Cohen's $d=.80$. Main effects and interaction involving object orientation did not reach significance $(F \mathrm{~S}=2.49, p>.131)$. Lastly, a two-way repeated-measures ANOVA with object orientation (horizontal, vertical) and object type (invalid same-object, invalid different-object) as within-subjects factors was conducted for both accuracy and RT to examine object-based effects, and no main effects or interactions for both RT and accuracy were significant, $(F \mathrm{~s}$ $<1.52$, $p \mathrm{~s}>.23$ ). To demonstrate support for the null hypothesis, the data were examined by calculating a Bayes factor (BF), comparing the fit of the data under the null and alternative hypotheses. For accuracy, an estimated $\mathrm{BF}_{01}$ suggested that the data were 2.49:1 in favor of the null hypothesis and for $\mathrm{RT}$, an estimated $\mathrm{BF}_{01}$ suggested that the data were 2.51:1 in

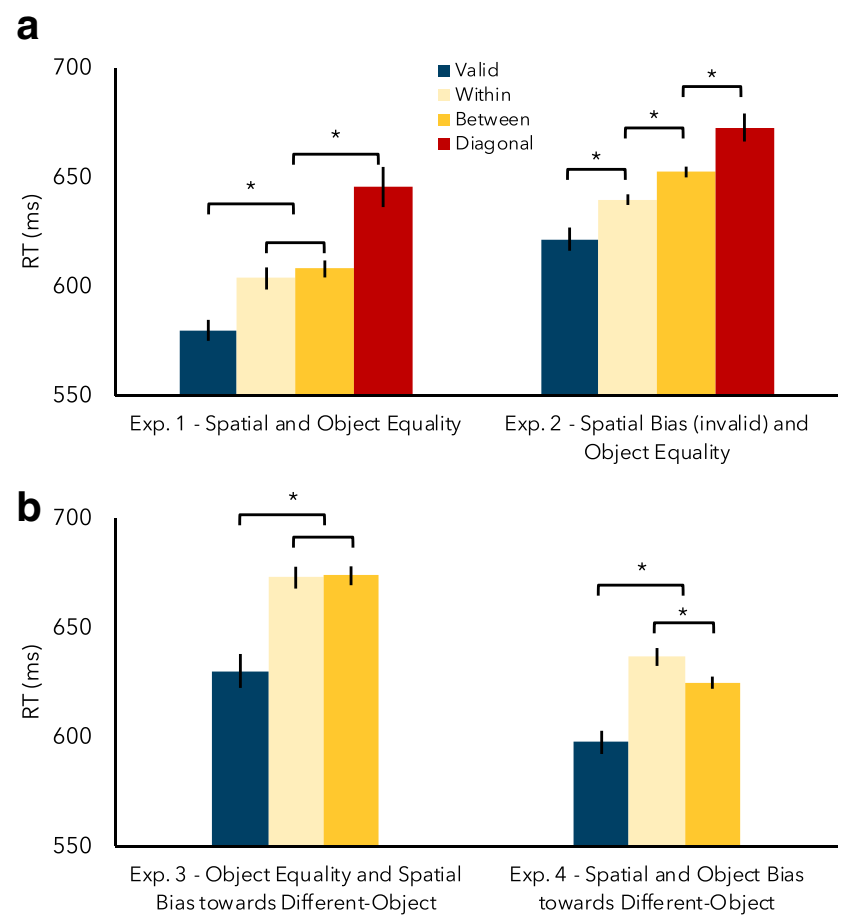

Fig. 2 Response time (RT) results for Experiments 1 and 2 (A) and Experiments 3 and 4 (B). Error bars represent \pm 1 standard error of the mean corrected for within-subjects (Morey, 2008). * Indicates significance at $\mathrm{p}<.05$ 
favor of the null hypothesis. In other words, the accuracy data were 2.49 times more likely to occur under the model without including an object-based effect than one with it and 2.51 for the RT data.

Although the results of Experiment 1 demonstrate that attention is driven only by spatial information when target appearance is equally distributed across two objects, a prior study (Goldsmith \& Yeari, 2003) has demonstrated an object-based attentional benefit using the same target probabilities. The main reason behind this discrepancy can be found in the different stimulus-onset asynchronies (SOAs) used in either studies. In the Goldsmith and Yeari (2003) study, the cue-target SOA was $150 \mathrm{~ms}$, shorter than the $350 \mathrm{~ms}$ used in the current experiment. Using the two-rectangle paradigm, Drummond and Shomstein (2010) implemented a cue that informed the participant that the target would appear with $100 \%$ certainty in either the sameobject or different-object location. Additionally, the SOA between cue and target was manipulated between 200, 400, or $600 \mathrm{~ms}$. At a short SOA (200 ms), an object-based effect was observed while no object-based effect was observed in the longer SOAs. This demonstrated that when given enough time, participants were able to apply knowledge of the certain target location. Thus, given enough time, and when an alternate strategy is present, attentional selection is not guided by objectbased representations. Based on the finding of this study, it makes sense that an object-based effect was observed in the study of Goldsmith and Yeari (2003) while no object-based effect was observed in the current study.

Experiment 1 examined whether object representations guide attention when target appearance is equally distributed across objects. Although the results provided evidence for faster and more accurate target identification in the cued location than any other spatially invalid conditions, demonstrating the robustness of spatial attention, no object-based effect was observed. While a null result should not be used as evidence for the absence of the effect, the findings suggest that object representations do not automatically influence attentional allocation. When the object representation does not contribute to reducing uncertainty, attention is solely guided by spatial representations.

\section{Experiment 2 - spatial bias (invalid) and object equality} Experiment 2 further investigated the interaction between spatial and object representations by biasing specific locations within objects while equating the probability of target appearance between the objects. If attentional allocation is only driven by spatial frequency, an equal benefit towards the two biased locations would be expected. If object representations contribute to attentional guidance, a benefit for targets in the invalid same-object location over the invalid different-object location would be expected. Lastly, if object representations do not contribute to reducing uncertainty, results identical to that of Experiment 1 were expected.
Two participants (average accuracy: $82.56 \%$ ) and $.91 \%$ of trials were removed from the analysis leaving a total of 24 participants in the final analysis. A repeated-measures ANOVA with object orientation (horizontal, vertical) as a between-subject factor and spatial distance (valid, invalid, diagonal) as a within-subject factor on accuracy did not reveal any significant main effect or interaction, $(F \mathrm{~s}<1.36, p \mathrm{~s}>.26)$. The same analysis on RTs revealed a significant main effect of spatial distance, $F(2,44)=17.15, \mathrm{p}<.001, \eta_{\mathrm{p}}^{2}=.44$. Planned $t$-tests revealed that participants were faster at responding to the target in the valid $(M=621.66 \mathrm{~ms}, \mathrm{SE}=5.32)$ than the invalid $(M=646.03, \mathrm{SE}=2.75,[t(23)=4.76, p<.001$, Cohen's $d=.97]$ and the diagonal trials $(M=672.79, \mathrm{SE}=$ 6.42), $[\mathrm{t}(23)=4.51, p<.001$, Cohen's $d=.92]$. Participants were also significantly faster at responding to the target in the invalid than in the diagonal trials, $t(23)=3.14, p=.014$, Cohen's $d=.64$. No other main effects or interaction reached significance $(F \mathrm{~s}<1, p \mathrm{~s}>.40)$.

A two-way repeated-measures ANOVA, with object type (invalid same-object, invalid different-object) as a withinsubject factor and object orientation (horizontal, vertical) as a between-subject factor was conducted for both accuracy and RT to examine object-based effects. While the accuracy ANOVA did not reveal any significant main effect or interaction $(F \mathrm{~S}<2.86, p \mathrm{~s}>.10)$, there was a significant main effect of object type for $\operatorname{RT}\left(F(1,22)=25.68, p<.001, \eta^{2}{ }_{p}=.54\right)$ with participants showing faster RTs in the invalid same-object $(M$ $=639.75, S E=2.18$ ) than in the invalid different-object location $(M=656.99, S E=2.83)$.

Experiment 2 replicated the results of Experiment 1, demonstrating that attention is driven by spatial representations. Interestingly, the results suggest that object representations guide attentional allocation when target frequency is equally biased towards the invalid locations. If attention was solely influenced by spatial frequency, an equal benefit towards the two biased locations would be expected. However, although the target appeared in both invalid locations equiprobably, responses toward the invalid same-object location were faster than the invalid different-object location. This suggests that object representations influence attentional allocation when there is probabilistic imbalance or uncertainty (some locations are more likely to contain a target than others). In Experiment 1 , when the cue was uninformative of target location and each location was equally likely to contain a target, attentional selection was driven exclusively by spatial representations. However, after introducing probabilistic inequities in Experiment 2, spatial and object representations together guided attention.

Experiment 3 - object equality and spatial bias towards different-object The results from Experiments 1 and 2 demonstrate that object-based effects are present only when there are probabilistic imbalances. If, however, spatial probabilities are 
what solely determine attentional priority, it is possible that attentional benefit would be maximized towards a location if there is enough bias. In Experiment 3, the target was most likely to appear in the invalid different-object location while the remaining half of the trials were equally distributed between the valid and invalid same-object location. If attention is guided by spatial probability, we would expect the most attentional benefit in the invalid different-object location. However, if attention is guided by object representations, a stronger benefit towards the invalid same-object location was expected.

One participant (average accuracy: $55.78 \%$ ) and $.30 \%$ of trials were excluded from the analysis, leaving a total of 17 participants. A two-way repeated-measures ANOVA with object orientation (horizontal, vertical) as a between-subject factor and spatial distance (valid, invalid) as a within-subject factor was conducted for both accuracy and RT. The ANOVA on accuracy did not reveal any significant main effect or interaction $\left(F_{\mathrm{s}}<3.62, p \mathrm{~s}>.07\right)$, while for RT, there was a significant main effect of spatial distance, $F(1,15)=$ $12.96, p=.003, \eta_{\mathrm{p}}^{2}=.46$. Planned paired $t$ tests revealed that participants were significantly faster at responding in the valid $(M=630.27 \mathrm{~ms}, S E=7.76)$ than the invalid trials $(M=$ 673.81, $S E=4.60), t(16)=3.67, p=.002$, Cohen's $d=.89$. To test object-based effects, another repeated-measures ANOVA with object orientation (horizontal, vertical) as a between-subject factor and object type (invalid same-object, invalid different-object) as a within-subject factor was conducted for both accuracy and RT, but no main effect or interaction reached significance $\left(F_{\mathrm{S}},<2.91 \mathrm{ps}>.10\right)$. To demonstrate support for the null hypothesis, the Bayes factor (BF) for both accuracy and RT was calculated, comparing the fit of the data under the null and alternative hypotheses. An estimated $\mathrm{BF}_{01}$ demonstrated that the data were 5.63:1 in favor of the null hypothesis for accuracy and 3.01:1 in favor of the null hypothesis for RT. In other words, the data were 5.63 times more likely to occur under the model without including an object-based effect than one with it for accuracy and 3.01 times for RT.

Experiment 3 again showed a significant validity effect demonstrating the robustness of spatial influence. Although the target was twice as likely to appear in the invalid different-object location than the invalid same-object location, there was no observed attentional benefit. This result suggests that attentional guidance is not solely dependent on spatial probability, but is constantly factoring in object contribution as well. While a specific location is biased, since the target can appear equally in both objects, object representations do not help reduce uncertainty and thus do not influence attention. Another possibility is that the probability difference between the invalid same-object and the invalid different-object conditions was not significant enough to warrant a bias towards a specific location.
Experiment 4 - spatial and object bias towards different-object The results of Experiment 3 suggested that even though a specific location was biased, attentional allocation is mainly driven by spatial location. However, although the target was twice as likely to appear in the invalid different-object location than any other location, there was no observed benefit of this probabilistic imbalance. In Experiment 4, we tested whether a greater bias toward the invalid different-object location would influence attentional guidance in favor of the uncued other object.

No participants and $.41 \%$ of trials were excluded from the analysis for a total of 25 participants. A repeated-measures ANOVA with object orientation (horizontal, vertical) as between-subjects factor and spatial distance (valid, invalid) as within-subjects factor was conducted for both accuracy and RT to measure space-based effect. The ANOVA on accuracy did not reveal any significant main effect or interaction $(F \mathrm{~s}<2.34, p \mathrm{~s}>.13)$, there was a significant main effect of spatial distance for RT, $F(1,23)=15.39, p<.001, \eta_{p}^{2}=.40$; participants were significantly faster at responding in the valid $(M=597.59 \mathrm{~ms}, S E=5.31)$ than the invalid trial $(M=627.21$, $S E=3.66$ ). Another repeated-measures ANOVA with object orientation (horizontal, vertical) as a between-subjects factor and object type (invalid same-object, invalid different-object) as a within-subject factor was conducted for both accuracy and RT. ANOVA on accuracy did not reveal any significant main effect of object type $(F=.04, p=.849)$, but for RT, there was a significant main effect of object type, $F(1,23)=6.35, p$ $=.019, \eta_{p}^{2}=.22$, with faster target identification in the invalid different-object $(M=624.90, S E=2.78)$ compared to the invalid same-object location $(M=636.66, S E=2.46)$.

In Experiment 4, target appearance was biased towards both the invalidly cued object as well as the invalid different-object location. An attentional benefit towards the biased object and location was observed, on top of the spatial validity effect, suggesting that spatial probabilities can be prioritized over object representations, resulting in a "reversed" object-based effect.

Across-experiment ANOVA To examine whether the magnitude of the object-based effect differed between experiments, the object-based effect (invalid different-object - invalid same-object) for RT was calculated for all experiments and a one-way ANOVA was conducted with experiment as a between-subject factor, revealing a significant difference across experiments, $F(1,82)=8.89, p<.001, \eta_{p}^{2}=.25$ (Fig. 3). A series of planned t-tests revealed that the objectbased effect in Experiment 2 and the reversed object-based effect in Experiment 4 were significantly different from each other, $[t(82)=5.13, p<.001]$ and the other two experiments ( $t \mathrm{~s}>2, p \mathrm{~s}<.05$ ). A series of more conservative Tukey post hoc contrasts revealed that the reversed object-based effect in Experiment 4 was again significantly different from the 


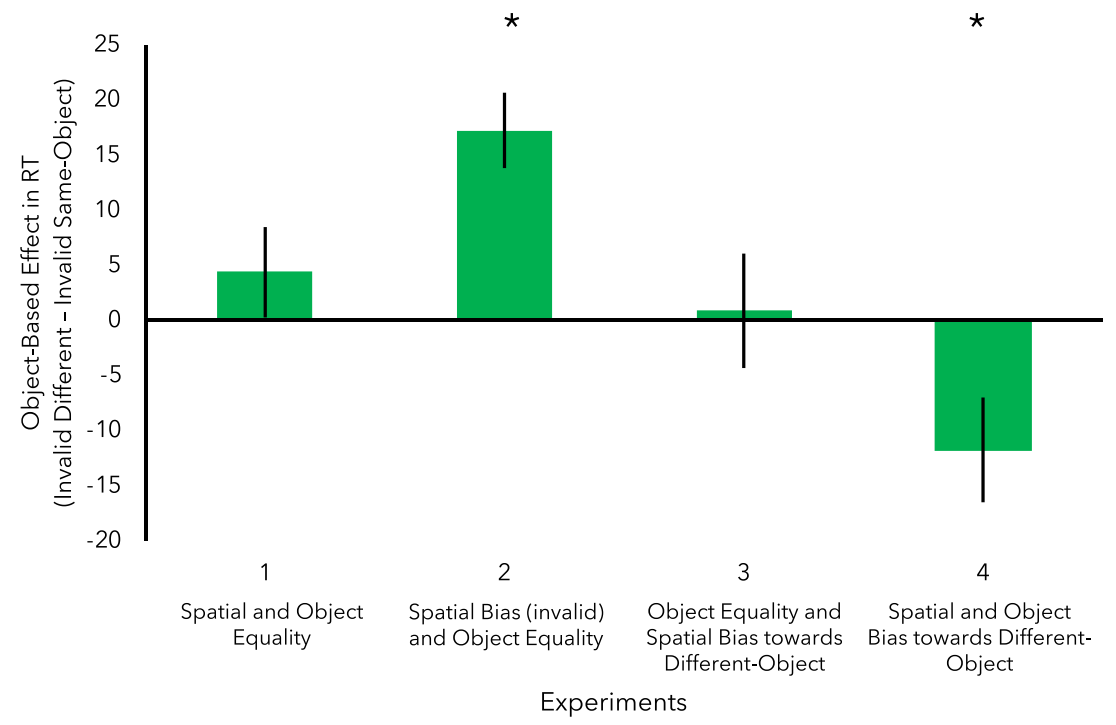

Fig. 3 Object-based effects in response time (RT) across all experiments. Error bars represent \pm 1 standard error of the mean

object-based effect in Experiment $2[t(82)=5.13, p<.001$, Cohen's $d=1.45]$ and the object-based effect in Experiment 1 $[t(82)=2.72, p=.039$, Cohen's $d=.78]$. No other differences were significant, $p \mathrm{~s}>.05$. These results demonstrate that when the target is equally biased towards the two invalid locations (Experiment 2), the attentional system relies on object representations to reduce the uncertainty of target location, and greater priority is designated to the within-object location over the equidistant between-object location. However, when target appearance is biased towards a different object (Experiment 4), the cue becomes informative of target location and reduces uncertainty, thus demonstrating an attentional benefit towards the unattended object.

\section{General discussion}

Over four experiments, we demonstrated that the influence object representations have on attentional guidance interacts with spatial probabilities. While objects bias attention, the contribution of objects can be replaced by spatial biases. Experiment 1 demonstrated that attention is guided by spatial information if the target is equally distributed across two objects. Experiment 2 equally biased the invalid same-object and invalid different-object locations to examine how object representations and spatial frequency interact and demonstrated that objects guide attentional allocation if used to prioritize between two equally biased locations. In Experiment 3, the invalid different-object location was biased while maintaining equal probability between objects to test whether attention is mainly guided by spatial frequency, demonstrating that spatial probability does not solely guide attention, but that object contribution is constantly influential as well. Lastly, Experiment 4 examined whether a bias towards the non- cued object can guide attention and provided evidence that if given enough discrepancy between locations, attention can be biased towards a non-cued object.

These findings are compatible with studies aiming to dissociate spatial biases and object-based effects. For instance, studies have failed to find an attentional benefit from object representations when a spatial cue is $100 \%$ informative of target location (Drummond \& Shomstein, 2010) as well as in the absence of a spatial cue (i.e., without a bias towards any object) (Donovan et al., 2017), providing evidence against automaticity of object-based attention. Recently, a study aiming to tease apart location-based and object-based cueing demonstrated that informative location-based cues resulted in a spatial cueing effect and informative object-based cues led to an object-based effect (Chou \& Yeh, 2018). However, when the object-based cues were informative (Experiments 1 and 3), target probability was biased towards the cued-object, making it difficult to ascertain whether object-based effects were the result of this probability imbalance or object representation. By parametrically manipulating spatial probabilities between the cue and target, the current study adds evidence that object representations do not automatically constrain attentional allocation. When a spatial cue is present but is uninformative of target location, attentional allocation is solely dictated by spatial locations. However, in the presence of a spatial bias towards two equidistant locations in either object, object representations contribute to attentional selection. Lastly, attention can be biased towards an uncued object if enough spatial bias is present.

Several theories have been developed to elucidate the underlying mechanism of the object-based effect, such as the attentional spreading, attentional prioritization, and uncertainty hypothesis. The attentional spreading hypothesis proposes that attention automatically spreads throughout a local area 
from the cued location to other non-cued locations, and that this spread respects object boundaries (Avrahami, 1999; Chen \& Cave, 2006, 2008; Ho, 2011). Thus, the object-based effect is the result of an automatic sensory enhancement that occurs within an object boundary. While this theory provides a clear explanation for the distance effect from spatial cueing paradigms, it fails to explain how top-down attentional biases incorporate scene segmentation and object boundaries (Shomstein, 2012).

The attentional prioritization hypothesis (Drummond \& Shomstein, 2010, 2013; Shomstein \& Yantis, 2002, 2004) directly challenges the automaticity assumption, and argues that object-based effects are the result of changes in attentional priority settings for possible target locations. It argued that priorities, and by extension object-based effects, are driven by the amount of spatial uncertainty regarding the upcoming target's position. For instance, Shomstein and Yantis (2002) observed object-based effects when the spatial location of the target was unpredictable, but this object-based attentional benefit was modulated by target location uncertainty; when the target location was $100 \%$ certain, no object-based effects were observed. If objects automatically constrain attentional allocation, an object-based effect should be expected regardless of the target certainty. Thus, the authors proposed that attentional priority is assigned in such a manner that when a cue predicts the upcoming spatial location, with certainty, other representations (e.g., object boundaries) are considered irrelevant and filtered out. However, when a cue does not predict the upcoming spatial location of the target with certainty, all available representations will be utilized to guide attention, including both space and object representations (Drummond \& Shomstein, 2010, 2013).

The uncertainty hypothesis (Shomstein, 2012), a more detailed expansion of the attentional prioritization hypothesis (Drummond \& Shomstein, 2010, 2013; Shomstein \& Yantis, 2002, 2004), posits that the goal of the attentional system is to reduce overall uncertainty in the environment. In highly uncertain situations (e.g., upcoming target location is unknown), other available representations that reduce the level of uncertainty (e.g., features, objects) constrain attentional allocation. However, in highly certain situations (e.g., upcoming target location is known), only the spatial representation constrains attentional allocation. Thus, higher attentional priority is assigned to spatial locations within an already attended object, if and only if, no other strategy is available. A critical element of the uncertainty hypothesis is that uncertainty is not restricted to space, but is generalizable across all attention-orienting elements (Shomstein, 2012). While other accounts that provide a mechanistic explanation exist, such as the attentional focusing (Goldsmith \& Yeari, 2003) and attentional shifting (Lamy \& Egeth, 2002) hypotheses, the focus here will mainly be between the attentional spreading and uncertainty hypotheses.
The evidence derived from the current study adds support to the uncertainty hypothesis over the attentional spreading hypothesis. Object contribution to attentional selection was modulated by the amount of spatial probability imbalance towards a specific object or location. If object representations automatically influence attentional allocation, all four experiments from the current study should have exhibited objectbased effects. However, an object-based effect was only present when both invalid same-object and invalid between-object was biased, suggesting that object representations influence attentional allocation when target appearance is uncertain. Object representations do not automatically constrain attentional allocation as predicted by the attentional spreading hypothesis, but rather are part of many representations in the environment that the attentional system can rely on to reduce uncertainty. These findings dovetail with accumulating evidence showing that object representations do not automatically guide attention, but are one of many factors in the environment that reduce uncertainty (Drummond \& Shomstein, 2010, 2013; Shomstein, 2012; Shomstein \& Johnson, 2013; Shomstein \& Yantis, 2002, 2004).

Open Practices Statement The data and materials for all experiments are available from the author upon request. None of the experiments were preregistered.

\section{References}

Avrahami, J. (1999). Objects of attention, objects of perception. Perception \& Psychophysics, 61(8), 1604-1612. doi:https://doi. org/10.3758/bf03213121

Behrmann, M., Zemel, R. S., \& Mozer, M. C. (1998). Object-based attention and occlusion: evidence from normal participants and a computational model. J Exp Psychol Hum Percept Perform, 24(4), 1011-1036. Retrieved from https://www.ncbi.nlm.nih.gov/pubmed/ 9706708

Carrasco, M. (2011). Visual attention: the past 25 years. Vision Res, 51(13), 1484-1525. doi:https://doi.org/10.1016/j.visres.2011.04.012

Chen, Z., \& Cave, K. R. (2006). Reinstating object-based attention under positional certainty: the importance of subjective parsing. Percept Psychophys, 68(6), 992-1003. Retrieved from https://www.ncbi. nlm.nih.gov/pubmed/17153193

Chen, Z., \& Cave, K. R. (2008). Object-based attention with endogenous cuing and positional certainty. Percept Psychophys, 70(8), 14351443. doi:https://doi.org/10.3758/PP.70.8.1435

Chou, W. L., \& Yeh, S. L. (2018). Dissociating location-based and object-based cue validity effects in object-based attention. Vision Res, 143, 34-41. doi:https://doi.org/10.1016/j.visres.2017.11.008

Donovan, I., Pratt, J., \& Shomstein, S. (2017). Spatial attention is necessary for object-based attention: Evidence from temporal-order judgments. Attention, Perception, \& Psychophysics, 79(3), 753-764. doi: https://doi.org/10.3758/s13414-016-1265-6

Drummond, L., \& Shomstein, S. (2010). Object-based attention: shifting or uncertainty? Attention, Perception, \& Psychophysics, 72(7), 1743-1755. doi:https://doi.org/10.3758/APP.72.7.1743 
Drummond, L., \& Shomstein, S. (2013). The timecourse of space- and object-based attentional prioritization with varying degrees of certainty. Front Integr Neurosci, 7, 88. doi:https://doi.org/10.3389/ fnint.2013.00088

Duncan, J. (1984). Selective attention and the organization of visual information. J Exp Psychol Gen, 113(4), 501-517. doi:https://doi.org/ 10.1037/0096-3445.113.4.501

Egly, R., Driver, J., \& Rafal, R. D. (1994). Shifting visual attention between objects and locations: evidence from normal and parietal lesion subjects. J Exp Psychol Gen, 123(2), 161-177. Retrieved from https://www.ncbi.nlm.nih.gov/pubmed/8014611

Eriksen, B. A., \& Eriksen, C. W. (1974). Effects of noise letters upon the identification of a target letter in a nonsearch task. Perception \& Psychophysics, 16(1), 143-149. doi:https://doi.org/10.3758/ bf03203267

Faul, F., Erdfelder, E., Lang, A.-G., \& Buchner, A. (2007). G*Power 3: A flexible statistical power analysis program for the social, behavioral, and biomedical sciences. Behavior Research Methods, 39(2), 175191. doi:https://doi.org/10.3758/bf03193146

Geng, J. J., \& Behrmann, M. (2002). Probability cuing of target location facilitates visual search implicitly in normal participants and patients with hemispatial neglect. Psychol Sci, 13(6), 520-525. doi:https:// doi.org/10.1111/1467-9280.00491

Goldsmith, M., \& Yeari, M. (2003). Modulation of object-based attention by spatial focus under endogenous and exogenous orienting. $J$ Exp Psychol Hum Percept Perform, 29(5), 897-918. doi:https://doi.org/ 10.1037/0096-1523.29.5.897

Ho, M. C. (2011). Object-based attention: sensory enhancement or scanning prioritization. Acta Psychol (Amst), 138(1), 45-51. doi:https:// doi.org/10.1016/j.actpsy.2011.05.004

Kravitz, D. J., \& Behrmann, M. (2011). Space-, object-, and feature-based attention interact to organize visual scenes. Attention, Perception, \& Psychophysics, 73(8), 2434-2447. doi:https://doi.org/10.3758/ s13414-011-0201-z

Lamy, D., \& Egeth, H. (2002). Object-based selection: the role of attentional shifts. Percept Psychophys, 64(1), 52-66. Retrieved from https://www.ncbi.nlm.nih.gov/pubmed/11916302

Marino, A. C., \& Scholl, B. J. (2005). The role of closure in defining the "objects" of object-based attention. Percept Psychophys, 67(7), 1140-1149. Retrieved from https://www.ncbi.nlm.nih.gov/ pubmed/16502836

Moore, C. M., Yantis, S., \& Vaughan, B. (1998). Object-based visual selection: Evidence from perceptual completion. Psychological Science, 9(2), 104-110. doi:https://doi.org/10.1111/1467-9280. 00019

Morey, R. D. (2008). Confidence Intervals from Normalized Data: A correction to Cousineau (2005). Tutorials in Quantitative Methods for Psychology, 4(2), 61-64. doi:https://doi.org/10.20982/tqmp.04. 2.p061

Müller, N. G., \& Kleinschmidt, A. (2003). Dynamic interaction of objectand space-based attention in retinotopic visual areas. $J$ Neurosci,
23(30), 9812-9816. Retrieved from https://www.ncbi.nlm.nih.gov/ pubmed/14586009

Nah, J. C., Neppi-Modona, M., Strother, L., Behrmann, M., \& Shomstein, S. (2018). Object width modulates object-based attentional selection. Attention, Perception, \& Psychophysics. doi:https:// doi.org/10.3758/s13414-018-1530-y

Posner, M. I. (1980). Orienting of attention. Quarterly journal of experimental psychology, 32(1), 3-25.

Posner, M. I., Snyder, C. R., \& Davidson, B. J. (1980). Attention and the detection of signals. J Exp Psychol, 109(2), 160-174. Retrieved from https://www.ncbi.nlm.nih.gov/pubmed/7381367

Robertson, L. C., \& Kim, M. S. (1999). Effects of perceived space on spatial attention. Psychological Science, 10(1), 76-79. doi: https:// doi.org/10.1111/1467-9280.00110

Rock, I., \& Gutman, D. (1981). The effect of inattention on form perception. J Exp Psychol Hum Percept Perform, 7(2), 275-285. doi: https://doi.org/10.1037//0096-1523.7.2.275

Shomstein, S. (2012). Object-based attention: strategy versus automaticity. Wiley Interdiscip Rev Cogn Sci, 3(2), 163-169. doi:https://doi. org/10.1002/wcs. 1162

Shomstein, S., \& Behrmann, M. (2006). Cortical systems mediating visual attention to both objects and spatial locations. Proc Natl Acad Sci U S A, 103(30), 11387-11392. doi:https://doi.org/10.1073/pnas. 0601813103

Shomstein, S., \& Behrmann, M. (2008). Object-based attention: strength of object representation and attentional guidance. Percept Psychophys, 70(1), 132-144. doi:https://doi.org/10.3758/PP.70.1. 132

Shomstein, S., \& Johnson, J. (2013). Shaping attention with reward: effects of reward on space- and object-based selection. Psychol Sci, 24(12), 2369-2378. doi:https://doi.org/10.1177/ 0956797613490743

Shomstein, S., \& Yantis, S. (2002). Object-based attention: sensory modulation or priority setting? Percept Psychophys, 64(1), 41-51. Retrieved from https://www.ncbi.nlm.nih.gov/pubmed/11916301

Shomstein, S., \& Yantis, S. (2004). Configural and contextual prioritization in object-based attention. Psychon Bull Rev, 11(2), 247-253. Retrieved from https://www.ncbi.nlm.nih.gov/pubmed/15260189

Theeuwes, J., Mathot, S., \& Kingstone, A. (2010). Object-based eye movements: the eyes prefer to stay within the same object. Attention, Perception, \& Psychophysics, 72(3), 597-601. doi: https://doi.org/10.3758/APP.72.3.597

Watson, S. E., \& Kramer, A. F. (1999). Object-based visual selective attention and perceptual organization. Perception \& Psychophysics, 61(1), 31-49. doi:https://doi.org/10.3758/ bf03211947

Watt, R. J. (1988). Visual processing: Computational, psychophysical, and cognitive research: Psychology Press.

Publisher's note Springer Nature remains neutral with regard to jurisdictional claims in published maps and institutional affiliations. 